\title{
Interactive comment on "WoSIS: Serving standardised soil profile data for the world" by Niels H. Batjes et al.
}

\section{A. Samuel-Rosa (Referee) \\ alessandrosamuelrosa@gmail.com}

Received and published: 29 November 2016

ISRIC World Soil Information is long known for its efforts for collecting, organising and serving quality soil data and information for the international community. Its latest enterprise, the World Soil Information Service (WoSIS), comes as the natural outcome of decades of responsible and sound scientific work. This is made very clear in this paper - the reason why I have only a few comments and questions.

The paper successfully describes in a very clear and concise way the procedures adopted by ISRIC for standardising, harmonising and serving global soil profile data. These seem to be the most time consuming in house tasks when building a truly usable soil database and the authors devoted most of their efforts to their description in this paper. However, I think that the paper - and WoSIS - would benefit from more details 
on the safeguarding step. The way I understand it, this first step of safeguarding soil data involves establishing and maintaining a close and lasting collaboration with soil data providers. Collaboration with institutions from different countries usually involve a series of bureaucratic - and political - decisions. In the case of WoSIS, I believe that data providers would be concerned about issues relating to intellectual property, user rights, and how they can benefit from contributing soil data. Thus, the questions: How does ISRIC approach potential data providers? What are the strategies used by ISRIC to deal with bureaucratic - and political - issues? How can an individual or organization contribute data for inclusion in WoSIS? What immediate benefits one can expect when contributing soil data?

In my experience, the lack of or poor georeferencing of soil profile data is a more serious problem for some soil data users, specially for geospatial applications, than the lack of harmonisation - provided one has the proper means for validating modelling outputs. In the current version of WoSIS, about $20 \%$ of the soil profiles do not have a quantitative georeference, i.e. numeric geographic coordinates. This is a hindrance to their usage. Thus, the question: What are the strategies planned for attributing numeric geographic coordinates to soil profiles that lack this sort of information?

\section{Specific comments}

Figure 2. Some text is unexpectedly underlined. Consider removing the underline or explaining its usage. Also, consider using a comma as a Thousands separator as it has been used in other parts of the text, e.g. the abstract.

Figure 4 (title): There is a typo in the figure title: "Location op soil profiles [...]" should read "Location of soil profiles [...]".

Figure 4: I suggest reducing the width of points so that the figure gives a more realistic view of under-represented regions of the world. In the current format it gives the false impression that, for example, Latin America (except for Paraguay and parts of Chile and Argentina) is already well represented and Latin Americans should not bother

Interactive

comment 
contributing more data. Also, consider presenting a summary table with the countries with the highest profile density (number of profiles per surface area) in WoSIS.

Finally, I must note that I agree with the comments and notes of Hugelius (2016), and would like to reinforce the suggestion of also serving all data in a singe large CSV file. In this case, for ease of use, I would suggest omitting the metadata, which should only be available in the single soil property files.

Interactive

comment

\section{Reference}

Hugelius, G. Interactive comment on "WoSIS: Serving standardised soil proïňĄle data for the world" by Niels H. Batjes et al.. Earth Syst. Sci. Data Discuss. 2016.

Interactive comment on Earth Syst. Sci. Data Discuss., doi:10.5194/essd-2016-34, 2016. 